American Journal of Biostatistics 1 (1): 67-74, 2010

ISSN 1948-9889

(C) 2010 Science Publications

\title{
Utilization and Modification of Point Sampling and Line Sampling in the Forestry Survey
}

\author{
Difei Zhang \\ Department of Natural Resource Ecology and Management, 008C AG Hall, \\ Oklahoma State University, Stillwater, OK, USA 74078
}

\begin{abstract}
Problem statement: In the practice of conventional point sampling and line sampling methods in the forestry survey, we often encounter problems such as boundary and hole problems. These problems could introduce bias in the results of forest sampling. Proper modifications are needed. Approach: This study developed novel probability computation approaches in the utilization and modification of horizontal point sampling and line sampling in the forestry inventory. It reviewed conventional point sampling and line sampling methods, identifies specific problems associated with actual forest sampling and provides modification solutions using probability computations. Results: By modifying the original point sampling and line sampling procedures, this study proposed novel solutions to these problems and provides better sampling methods with reduced bias in the forestry survey. Conclusion: In this study, only horizontal gauging for point sampling and line sampling was discussed. For the corresponding problems encountered in vertical gauging, the solutions are similar to the ones for the horizontal gauging sampling. These modifications had been presented with varying levels of complexity. To maintain a balance between precision and costs, modifications with an appropriate level of complexity may be selected.
\end{abstract}

Key words: Point sampling, line sampling, forest sampling, horizontal gauging, tree basal area

\section{INTRODUCTION}

The theoretical development of the point sampling and line sampling methods assume the land area being sampled has perfect geometric characteristics. There are several assumptions about the geometric characteristics of the study area, which may limit their utilizations in the forestry inventory survey:

- $\quad$ They ignore the case in which the sampling point or the sampling line is at the boundary. The theory assumes the sampling point or the sampling line is far enough from the boundaries to avoid the influence of boundaries. There are several previous approaches to this such as the mirage method (Schmid-Haas, 1982), Ducey's walk-through method (Ducey et al., 2004), Grosenbaugh's method (Grosenbaugh, 1979) and Beers (1977) method

- They assume that the plane of the study area is perfectly level. A solution to this is given in Avery and Burkhart (2001)

- They assume that the study area is a continuous plane without any "hole"

Due to these constraints, it is difficult to perform the theoretical point sampling and line sampling methods on a land area without introducing any bias. Proper modifications are needed. The first two problems as presented above have been solved and this study will address the modifications to solve the third problem addressed here: the boundary and hole problem. In addition, this study will propose a different solution to the boundary overlap problem compared with the walkthrough method proposed by Ducey et al. (2004).

Literature review: Point sampling is a commonly used method in forest inventory sampling (Oderwald and Jones, 1992). Horizontal point sampling theory states that the vertex of a constant angle whose sides are exactly tangent to a circular tree cross section will generate a huge imaginary ring on a level plane around the tree if the vertex is pivoted about the tree center. Horizontal angle-gauge with vertex at sample point tells observer point lies inside imaginary ring of one tree and outside the imaginary ring of another tree. The forester determines what trees are to be included in the sample by sighting through an angle gauge, such as a prism, also known as variable radius plot sampling, or simply prism sampling. The horizontal point samples can be located near stand boundaries where a portion of the sweep falls outside the stand. The boundary overlap can 
be corrected using the mirage method developed by Schmid-Haas (1982) and described by Beers (1977). The guidelines to be followed when the mirage method is applied in horizontal sampling have been explained by Husch et al. (1982) and Bertram et al. (2002). Overlapping occurs when the plot Radius (Ri) associated with any qualifying tree exceeds the shortest perpendicular distance (B) from the sample point to the boundary. If $\mathrm{Ri}>\mathrm{B}$ overlap exists, $\mathrm{Ri}$ may be determined using several equations or using diameter/plot radius ratios. Faham et al. (2008) case study in West Mazandaran showed sampling factors in the context of social forestry.

Point sampling can be used only to measure stand basal area over bark. If underbark basal area is required a method is needed to convert the overbark measurement to an underbark measurement. There are always trees for which the observer will find it difficult to judge whether they should or should not be included in the count. These will be trees which are very near the circumference of a circle being implicitly swept out by the observer. When such cases arise, the diameter of the tree at breast height over bark and the distance from its stem centre to the observer should be actually measured (West, 2009). Point-3P sampling, developed by Grosenbaugh $(1971,1979)$ is an efficient sampling design (Steber and Space, 1972; Van Hooser, 1978; Wood, 1982; Biggs et al., 1985; Mackisack and Wood, 1990). Van Hooser (1978) and Wood (1982) have also provided cost data by applying Point-3P sampling. Stamatellos (1992) compared six sampling designs (three Plot-3P, two Point-3P and one Plot-PPS) based on a cost-effectiveness index (sampling error squared $X$ cost). The two Point-3P designs were found to be more efficient.

Line sampling is an extension of the point sampling theory. It employs random-sample linesegments instead of sample points and the probability per unit-length of line that a particular tree will be selected is proportional to tree diameter (horizontal gauging) or to tree height (vertical gauging) instead of proportional to the square of these dimensions as in point-sampling. The angle-gauge is used to select sample trees on both sides of sample line- segments located at, random on the tract. Often continuous and parallel lines will be used; they are analogous to strips. At other times short discontinuous segments, analogous to rectangular plots, will be preferred. Horizontal line sampling using an angle gauge was originally developed by Strand in 1957 and its basic theory was outlined by Grosenbaugh (1958). Ducey et al. (2002) presented a straightforward modification of horizontal line sampling designed to facilitate inventory of features such as snags and cavity trees while remaining compatible with commonly employed sampling methods for the living overstory. This study used short segments for HLS and augmented the horizontal line sample by completing the angle gauge sweep around the end of the line, effectively adding half of a conventional horizontal point sample to each end of the line. This modification eliminated some of the practical difficulties encountered with traditional HLS and gave a straightforward way of estimating abundance, basal area, volume and other related attributes of snags and cavity trees in a forested tract. According to Ducey et al. (2002), the modified form of HLS is easy to implement in the field and appeared to be at least competitive with and in some cases better than, HPS for estimating stand parameters for snags and cavity trees. The fact that meaningful estimates can be obtained with sample sizes (number of line segment centers) comparable with the number of inventory points often used for timber inventories and other assessments of the living overstory is an additional advantage. Also, the method requires no extra equipment and uses basic techniques already familiar to many practitioners. Ducey et al. (2004) proposed another new walkthrough method for reducing boundary overlap bias that diminishes or eliminates the need to work outside the tract and accommodates irregular boundaries easily. Under typical conditions, the walkthrough method eliminates the boundary overlap bias associated with most objects near the border and reduces it for the remaining objects.

\section{MATERIALS AND METHODS}

When conducting a sample on a definite land area, foresters are primarily concerned with inventory attributes, which are to be obtained by summing up the individual values of that attribute on every tree in the land area sampled, for example the total number of trees or the total volume of the trees in the land area. On large areas, it is usually necessary to estimate these attributes by sampling.

The differences between point sampling and line sampling are in the methods they use to select trees and the formulas they use to calculate the probability of sampling the selected trees. After a tree is selected and its probability of selection is calculated, the computation of the estimated total value of the specific attribute in the sampled area is the same for both methods. We will discuss the steps for the estimation of the total value first.

Given a selected tree (i), its probability of selection is calculated as $P_{i}$ and the attribute of the selected tree we are interested in is sampled as $Y_{i}$ (for example, 
diameter, basal area and volume), an unbiased estimation of the total value of $\mathrm{Y}$ for the population in this study area would be $\mathrm{Y}_{\mathrm{i}} / \mathrm{P}_{\mathrm{i}}$. For all the selected trees, the unbiased sample-based estimation of the total value of $\mathrm{Y}$ for the population would be $\frac{1}{n} \sum_{i=0}^{m} \frac{y_{i}}{p_{i}}$ ( $m$ is the total number of the selected trees and $n$ is the total times we perform the random tree selection).

The point sampling method starts from a randomlyselected point in the land area of interest. From each point we use a predefined gauge-angle $\theta$ to select each sample tree and to calculate its associated $\mathrm{P}_{\mathrm{i}}$ (Fig. 1).

We assume the land area to be sampled is level and tree stem is vertical. On the plane that intersects a given tree at the breast height $(1.3 \mathrm{~m}$ above the ground $)$ and which is parallel to ground level, there is a gauge-angle of $\theta$ degrees whose vertex is the randomly selected sample point having a bisector which goes through the center of the tree. If edges of the gauge-angle intersect the tree's basal area (which is the cross-sectional area at breast height), the tree is selected and its probability $P_{i}$ is calculated as Eq. 1:

$$
\mathrm{P}=\frac{\mathrm{TBA}}{\left(\sin \frac{\theta}{2}\right)^{2} * \mathrm{~A}}=\frac{\mathrm{DBH}^{2} * \pi}{4 *\left(\sin \frac{\theta}{2}\right)^{2} * \mathrm{~A}}
$$

Where:

$$
\begin{aligned}
& \text { TBA }=\text { Tree Basal Area } \\
& \text { DBH }=\text { Diameter at Breast Height } \\
& \mathrm{A} \quad=\text { Area sampled } \\
& \Theta \quad=\text { The gauge angle }
\end{aligned}
$$

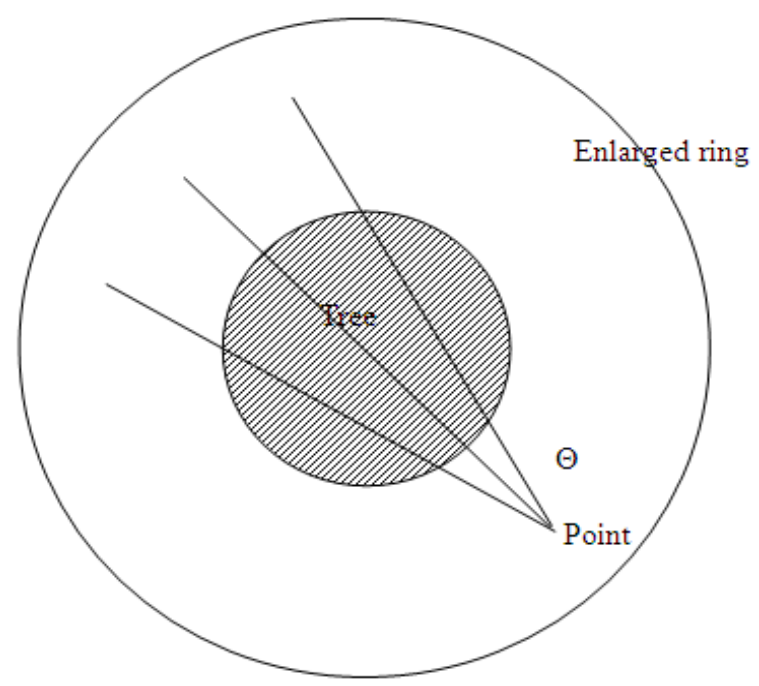

Fig. 1: Selecting trees in point sampling
The line sampling method is actually a combination of point sampling and the commonly used method of line-intersect sampling. In traditional lineintersect sampling, the diameter of the study object is used directly to calculate the probability of selection. In forest sampling, the comparatively low density of the forest does not allow the randomly generated line to intersect with enough trees to obtain a sufficiently low estimation variance. The line sampling method enlarges the tree diameter (at breast height) by using the gaugeangle, first introduced in the point sampling method. Given the gauge-angle $\theta$, the enlarged virtual tree diameter is $\mathrm{DBH}^{*} \csc (\theta / 2)$.

We assume the land area to be sampled is level and the tree stem is vertical. On a plane intersecting the tree at the breast height ( $1.3 \mathrm{~m}$ above the ground) and which is parallel to ground level, there is a gauge-angle $\theta$ of degrees whose vertex is located at the line and whose bisector goes through the tree center and is perpendicular to the line. If both edges of the gaugeangle intersect with the tree's basal area (which is the cross sectional area at the breast height), the tree is selected (Fig. 1) and its probability $\mathrm{P}_{\mathrm{i}}$ is calculated as Eq. 2:

$$
\mathrm{P}_{\mathrm{i}}=\frac{\mathrm{DBH} * \mathrm{~L}_{\mathrm{i}}}{\sin \frac{\theta}{2} * \mathrm{~A}}
$$

where, $\mathrm{L}_{\mathrm{i}}$ is the line length.

The point sampling method uses the gauge-angle to enlarge the sampling area standing for the selected tree from its basal area $\left(B_{i}\right)$ to $\frac{B_{i}}{\left(\sin \frac{\theta}{2}\right)^{2}}$. The line sampling also enlarges the tree's diameter at breast height by the factor $\frac{1}{\sin \frac{\theta}{2}}$. When the selected tree is far away from the study area boundaries, the enlarged virtual tree basal area or the tree's diameter will not exceed the of the land area being sampled. No change is needed to use theoretical sampling method in this case. But when the selected tree us located close to the boundaries of the land area being sampled, the enlarged tree basal area or the tree diameter will be projected beyond these boundaries (Fig. 2). Theoretically the part of the enlarged area or diameter which extends across the boundary of the land area being sampled will introduce bias into the sample results, although the bias may not very significant when the study area is large enough. 


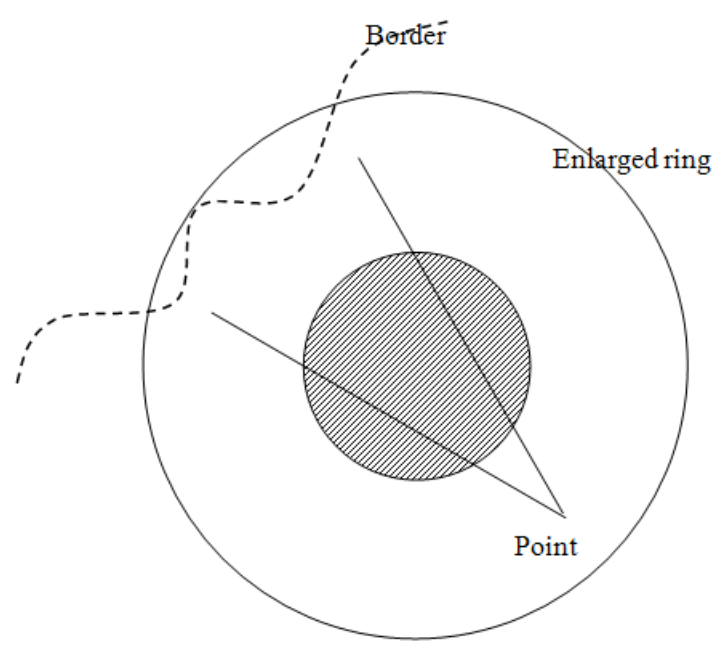

Fig. 2: Boundary overlap problem in point sampling

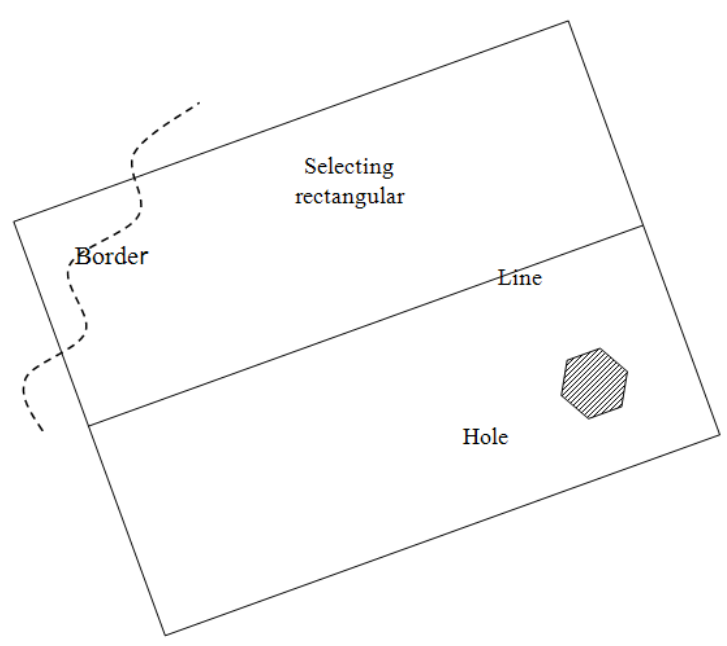

Fig. 3: Rectangle area bisected by the selected line in line sampling

Ducey et al. (2004) introduced a walkthrough method as a solution to the boundary overlap problem. They give a thorough discussion of a number of other approaches to the boundary overlap method such as the mirage method. Gregoire (1982) proved that the mirage method was unbiased under typical conditions with a straight boundary line, however, the mirage method cannot be applied unless it is possible to cross the boundary of the forested area being sampled to establish a mirage point. The walkthrough method proposed by Ducey et al. (2004) is unbiased and does not require travel outside the tract boundary. In this study, a different solution to the boundary overlap problem will be proposed.

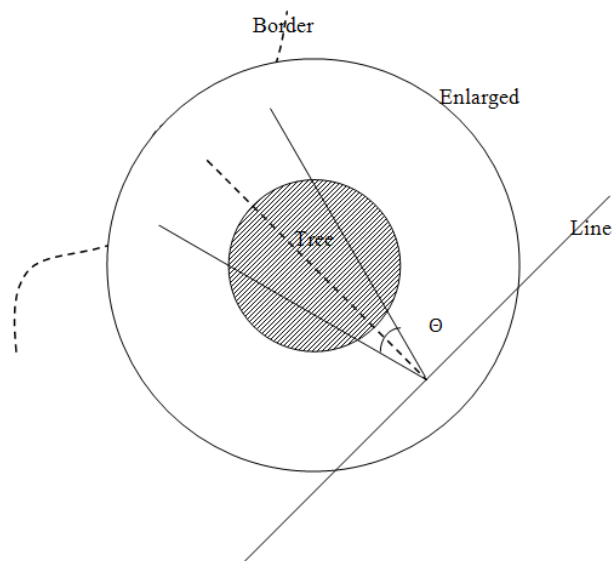

Fig. 4: Boundary overlap problem in line sampling

In the line sampling method, when a line is determined, the range used to determine whether a tree is selected or not is rectangular area that is bisected by the selected line. The length of the edge that is parallel to the selected line is equal to the length of that selected line. And the length of the edge that is perpendicular to that selecting line is $\frac{D \max }{\sin \frac{\theta}{2}}$. When this range exceeds the study area, the same bias is introduced as the one found in the point sampling method (Fig. 3 and 4).

\section{RESULTS AND DISCUSSION}

It is generally assumed that the land area to be sampled is a continuous range without any holes. However, there is always the possibility of the existence of holes in this land area. For example, there may be some small areas inside the study area, such as a small pond, a brook or a stream, where trees could not exist. Therefore these areas should be subtracted from the study area. In such a case, the land area sampled is not a contiguous range without holes. As in the case of the boundary overlap problem, when the enlarged tree basal area, enlarged tree diameter, or selecting range of the point or line, intersects or contains any of these holes, bias may occur if the hole area is not subtracted from the total land area being sampled (Fig. 5 and 6).

Because the way in which the bias is caused by the boundary and hole problems are very similar, the solutions for them are also similar. This solution requires adjustment of sample tree selection and corresponding adjustment of selection probabilities. We will discuss the solution for point sampling method first. 


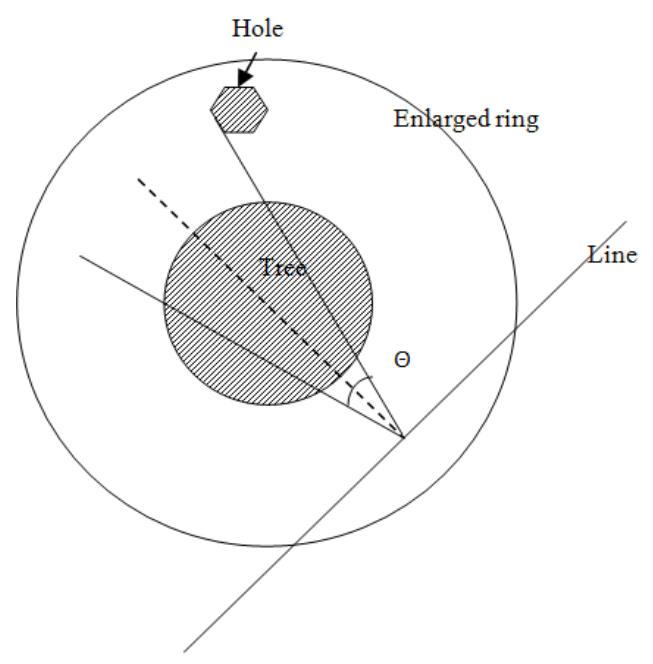

Fig. 5: Hole problem outside of the gauge angle in line sampling

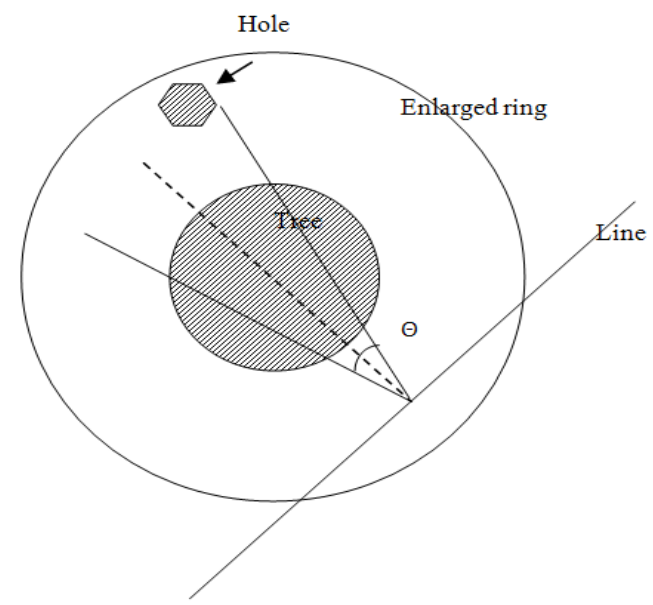

Fig. 6: Hole problem inside the gauge angle in line sampling

In the land area sampled, there is a maximum $\mathrm{D}_{\max }$ value for the tree diameter at breast height. On a random selected point $(j)$ given a constant gauge angle $(\theta)$, which is determined by the particular angle gauge being used, all trees that could be selected by this point (j) should be within a sector area $\left(A_{j}\right)$ whose center is that point, angle is $\varphi_{\mathrm{j}}$ and whose diameter is $\frac{\mathrm{D}_{\max }}{2 * \sin \frac{\theta}{2}}$. We use $\frac{D_{\max }}{2 * \sin \frac{\theta}{2}}$ to insure that the sector area does not contain any boundaries or special area (such as holes).

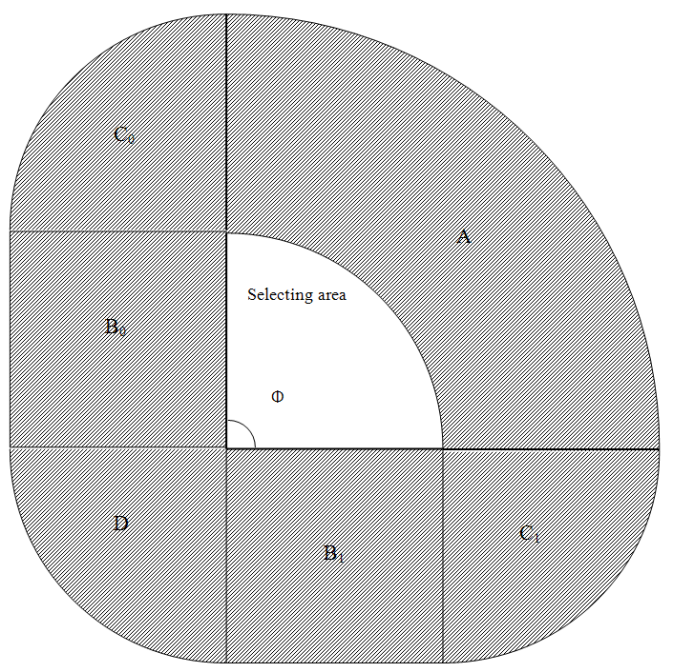

Fig. 7: Shadowed area around selecting area in point sampling

Every tree that could be sampled without introducing bias is selected. We use the magnitude of the angle $\left(\varphi_{\mathrm{j}}\right)$ as a parameter in the computation of the probability. Then we can adjust the degree of the angle $\left(\varphi_{\mathrm{j}}\right)$ to avoid intersecting with or containing any area outside the sampling area or whole area. When the degree of the angle $\left(\varphi_{\mathrm{j}}\right)$ is determined, the $P_{\mathrm{i}}$ can be calculated using the following equation.

$\mathrm{P}_{\mathrm{i}}=\frac{\phi_{\mathrm{j}} * \mathrm{TBA}}{360 *\left(\sin \frac{\theta}{2}\right)^{2} * \mathrm{~A}}=\frac{\phi_{\mathrm{j}} * \mathrm{DBH}^{2} * \pi}{360 * 4 *\left(\sin \frac{\theta}{2}\right)^{2} * \mathrm{~A}}$

When the angle $\left(\varphi_{\mathrm{j}}\right)$ is equal to $360^{\circ}$, the formula can be simplified to Eq. 4 :

$\mathrm{P}_{\mathrm{i}}=\frac{\mathrm{TBA}}{\left(\sin \frac{\theta}{2}\right)^{2} * \mathrm{~A}}=\frac{\mathrm{DBH}^{2} * \pi}{4 *\left(\sin \frac{\theta}{2}\right)^{2} * \mathrm{~A}}$

The result is the same as the original formula without considering the boundary and hole problem, which means that the randomly selected point is far away enough from the boundary or hole to avoid their biasing influence.

So the key point is to find the correct angle $\left(\varphi_{\mathrm{j}}\right)$. There are two constraints for selecting the angle $\left(\varphi_{\mathrm{j}}\right)$ (Fig. 7).

The sector area defined by the angle $\left(\varphi_{\mathrm{j}}\right)$ should not intersect with or contain any portion of a hole or area outside the boundary. Only trees in this area can be sampled. 


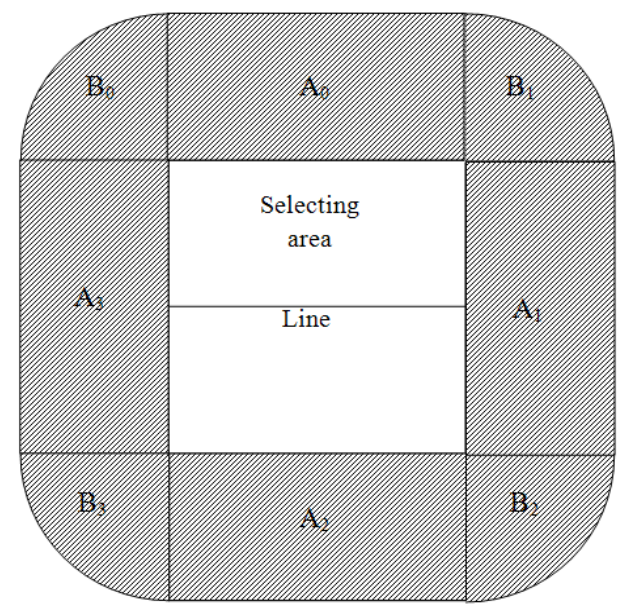

Fig. 8: Shadowed area around selecting area in line sampling

Figure 8 indicates a shadowed area surrounding the angle area. This shadowed area consists of four parts:

- Area A: it is narrow area with the width of $\frac{D_{\max }}{2 * \sin \frac{\theta}{2}}$.

Its inner radius is $\frac{D_{\max }}{2 * \sin \frac{\theta}{2}}$ and outer radius is $\frac{D_{\max }}{\sin \frac{\theta}{2}}$

- Two rectangular areas with dimension of $\frac{\mathrm{D}_{\max }}{2 * \sin \frac{\theta}{2}}$

long and $\frac{D_{\max }}{2 * \sin \frac{\theta}{2}}$ wide. One vertex of both of the

rectangles is the vertex of the angle and one longer edge of both of the rectangles is sitting on the edge of the angle

- Two sector areas with 90 degree angle and radius of $\frac{D_{\max }}{2 * \sin \frac{\theta}{2}}$. One edge of the angle is the shorter edge of one rectangle. The other rectangle is sitting on the bigger circular area

- One sector area with $\left(180-\varphi_{\mathrm{j}}\right)$ degree angle and radius of $\frac{D_{\max }}{2 * \sin \frac{\theta}{2}}$. Its two edges are the shorter edges on the two rectangles

This shadowed area is defined to make sure if the tree is located on the boundary of the selecting area, the enlarged tree basal area of any selected tree will not contain the part of any special area.

These two constraints are defined from a theoretical viewpoint. In the practical applications, the second constraint is not easy to employ because its geometric shape is complex for field implementation. The probability that a selected tree is located at the boundary of the selecting area and also has a diameter at breast height that is near the maximum value is relatively small. The second constraint can be omitted for practical convenience without introducing too much bias. We only need to determine the angle $\varphi_{\mathrm{j}}$ by the first constraint. Next we will discuss the solution for the line sampling method.

In the original line sampling method, the tree selecting area is a rectangle instead of a ring in the point sampling method. Just as we did for the point sampling method, we also put constraints on the line sampling selecting area to avoid the bias introduced by the special holes and boundary overlap.

Given a selected line (j) with length $\mathrm{L}_{\mathrm{j}}$, the gauge angle $(\theta)$ and the maximum tree diameter at breast height $D_{\max }$, the tree selection area is a rectangle with the dimensions of length $L_{i}$ and width $\frac{D_{\max }}{\sin \frac{\theta}{2}}$. We use $\frac{D_{\max }}{\sin \frac{\theta}{2}}$ to insure that, the enlarged tree basal area for the tree in the rectangular area does not contain holes or boundary overlap. When these trees are sampled, no bias will be introduced.

To avoid the possibility that the rectangular selection area intersects with or contains holes or overlaps the boundary, we adjust the length of the selecting line to change the size of the selection area. For the $\phi_{\mathrm{j}}$ parameter in the point sampling method, the key point is to find the proper $L_{j}$. Once the $L_{j}$ is determined.

The probability of a selected tree in the selecting area can be calculated using following formula (Eq. 5):

$$
\mathrm{P}_{\mathrm{i}}=\frac{\mathrm{DBH} * \mathrm{~L}_{\mathrm{j}}}{\sin \frac{\theta}{2} * \mathrm{~A}}
$$

The constraints for determining the length of the selecting line area are (Fig. 8):

- The area defined by the length $\left(\mathrm{L}_{\mathrm{j}}\right)$ should not intersect with or contain any hole or boundary overlap area. Only the trees in this area can be sampled with this particular line $\mathrm{j}$ 
- As shown in the Fig. 8, there are shadowed areas surrounding the rectangular area. The shadowed areas consist of four rectangular areas and four sector areas. The dimensions of these rectangular areas are $\frac{D_{\max }}{2 * \sin \frac{\theta}{2}}$ in width and $\frac{D_{\max }}{\sin \frac{\theta}{2}}$ in length. The sector areas are quarter of a disc with radius of $\frac{D_{\max }}{\sin \frac{\theta}{2}}$. The shadow area will not contain any special area or overlap with any boundary. The shadowed areas ensures if the tree is located on the boundary of the selecting area, its enlarged tree two basal area will not contain any part of the special area. We can simplify the shadowed areas to two rectangular areas with dimensions of $\frac{D_{\max }}{2 * \sin \frac{\theta}{2}}$ in width and $\frac{2 * D_{\max }}{\sin \frac{\theta}{2}}$ in length without introducing bias

Since the probability that a selected tree is located at the boundary of the selecting area and that the nearly maximum value of tree diameter at the breast height is relatively small, the second constraint can be ignored and its bias is omitted as well. By putting constraints on the tree selecting area, the boundary problem and hole problem can be solved theoretically. Even ignoring some constraints for practical convenience, the bias caused by boundary problem and hole problem can be reduced greatly. These approaches are designed for sampling land areas with irregular boundaries. For some special cases, such as the rectangular area, the solution can be even simpler. Defining peripheral zones is a simpler solution to the boundary problem in case of sampling a land area having rectangular boundaries (Grosenbaugh, 1958).

\section{CONCLUSION}

This study addresses the utilization and modification of horizontal point sampling and line sampling in the forestry inventory. It reviews conventional point sampling and line sampling methods and identifies problems associated with actual forest sampling. By modifying the original point sampling and line sampling procedures in some situations, this study proposes novel solutions to these problems and provides better sampling methods with reduced bias. In this study, only horizontal gauging for point sampling and line sampling methods is discussed. For the corresponding problems encountered in vertical gauging, the solutions are similar to the ones for the horizontal gauging sampling. These modifications have been presented with varying levels of complexity. To maintain a balance between precision and cost, modifications with an appropriate level of complexity may be selected.

\section{ACKNOWLEDGEMENT}

This study is approved for publication by Director of Oklahoma Agricultural Experiment Station in Oklahoma State University. It is funded by USDA CSREES grant project OKL02738, USDA Forest Service Southern research station grant and USDA Forest Service Federal Financial Assistance Research Award.

\section{REFERNCES}

Avery, T.E. and H.E. Burkhart, 2001. Forest Measurements. 5th Edn., McGraw-Hill, New York, ISBN: 10: 0073661767, pp: 480.

Beers, T.W., 1977. Practical corrections of a boundary overlap. Southern J. Applied For., 1: 16-18. http://www.ingentaconnect.com/content/saf/sjaf/19 77/00000001/00000001/art00007

Bertram, H., W.T. Beers and J.A. Kershaw, Jr., 2002. Forest Mensuration. 4th Edn., Wiley, New York, ISBN: 10: 0471018503, pp: 456.

Biggs, P.H., G.B. Wood, H.T. Schreuder and G.E. Brink, 1985. Comparison of point-model based and point-Poisson sampling for timber inventory in Jarrah Forest. Aust. For. Res., 15: 481-493. http://cat.inist.fr/?aModele $=$ afficheN\&cpsidt $=8126776$

Ducey, M.J., G.J. Jordan, J.H. Gove and H.T. Valentine, 2002. A practical modification of horizontal line sampling for snag and cavity tree inventory. Can. J. For. Res., 32: 1217-1224. DOI: 10.1139/X02-038

Ducey, M.J., J.H. Gove and H.T. Valentine, 2004. A walkthrough solution to the boundary overlap problem. For. Sci., 50: 427-435. http://www.ingentaconnect.com/content/saf/fs/200 4/00000050/00000004/art00003

Faham, E., A. Rezvanfar and T. Shamekhi, 2008. Analysis of factors influencing motivation of villagers' participation in activities of social forestry (the case study of West Mazandaran). Am. J. Agric. Biol. Sci., 3: 451-456. http://www.scipub.org/fulltext/AJAB/AJAB32451456.pdf 
Gregoire, T.G., 1982. Notes: The unbiasedness of the mirage correction procedure for boundary overlap. For. $\quad$ Sci., 28: 504-508. http://www.ingentaconnect.com/content/saf/fs/198 2/00000028/00000003/art00012

Grosenbaugh, L.R., 1958. Point-sampling and linesampling: Probability theory, geometric implications, synthesis. Southern Research Station. http://www.treesearch.fs.fed.us/pubs/2458

Grosenbaugh, L.R., 1971. STX 1-1 1-71 for dendrometry of multistage 3P samples. USDA Forest Service Publications.

Grosenbaugh, L.R., 1979. 3P sampling theory, examples and rationale. USDI Bur. Land Management Technology.

Husch, B., C.I. Miller and T.W. Beers, 1982. Forest Mensuration. 1st Edn., John Wiley and Sons, New York, pp: 402.

Mackisack, M.S. and G.B. Wood, 1990. Simulating the forest and the point-sampling process as an aid in designing forest inventories. For. Ecol. Manage., 38: 79-103. DOI: 10.1016/0378-1127(90)90087-R

Oderwald, R.G. and E. Jones, 1992. Sample sizes for point, double sampling. Can. J. For. Res., 22: 980-983. DOI: $10.1139 / \times 92-131$

Schmid-Haas, P., 1982. Sampling at the Forest Edge. In: Statistics in Theory and Practice: Essays in Honor of Bertil Matern, Ranneby, B. (Ed.). Swedish University of Agriculture and Sciences, Umea, Sweden, pp: 263-276.
Stamatellos, G.S., 1992. Efficiency of one-stage and two-stage sampling designs, the occasion of fixedarea sampling plots. Proceeding of Hellenic Forestry Association Annual Conference, Mar. 4-6, Hellenic Forestry Association, Research and Practice in Greek Forests, Kalamata, Greece, pp: 117-123.

Steber, G.D. and J.C. Space, 1972. New inventory system sweeping the South. J. For., 70: 76-79.

Van Hooser, D.D., 1978. Potential for 3P sampling in large-scale forest inventories. Proceeding of IUFRO Conference, June 18-26, 1978, Institute de Cercetari Si Amenjari Silvice, National Forest Inventory, Bucharest, Romania, pp: 52-59.

West, P.W., 2009. Tree and Measurement. 2nd Edn., Springer, New York, pp: 190.

Wood, B., 1982. Trial of point-3P sampling in an Australian hardwood forest. Proceedings of Biennial Meeting of Research Working Group No. 2, May 10-15, Australian Forestry Council, Hobart, pp: $1-14$. 